\title{
Trabajar el fragmento, ser la ruina. Lectura de un tríptico en Antropofaguitas de Gabriela Ponce Padilla
}

\author{
Working the fragment, being the ruin. \\ Reading of a triptych in Antropofaguitas, \\ by Gabriela Ponce Padilla

\section{María Auxiliadora Balladares Universidad San Francisco de Quito}

DOI: https://doi.org/10.32719/13900102.2018.44.5

Fecha de recepción: 8 enero 2018 Fecha de aceptación: 29 marzo 2018 


\section{RESUMEN}

La estética de lo fragmentario es la característica principal del relato "Caja Negra" (del libro Antropofaguitas, 2013), de Gabriela Ponce Padilla. Se trata de un relato compuesto de tres historias que narran la vida de tres mujeres ecuatorianas, en diferentes circunstancias históricas y sociales. El dispositivo de la caja negra es la imagen que une las tres historias que en términos formales replican los escritos, testimonios y diálogos que permiten reconstruir los últimos momentos de la vida de las protagonistas. La reflexión en torno a la ruina (de la mano del pensamiento de María Zambrano, Walter Benjamin y otros) nos permite entender las formas de resistencia desde donde se erigen los relatos de Ponce Padilla.

Palabras clave: Fragmento, ruina, tríptico, mujer, violencia patriarcal, violencia estatal, narrativa ecuatoriana, Gabriela Ponce Padilla.

\section{ABSTRACT}

The aesthetics of the fragmentary is the main characteristic of the story "Black Box" (from the book Antropofaguitas, 2013), by Gabriela Ponce Padilla. It is a narration composed of three stories that tell the lives of three Ecuadorian women in different historical and social circumstances. The black box is the device that puts together the three stories that, in terms of form, replicate the writings, testimonies and dialogues that allow reconstructing the last moments for the protagonists' lives. The reflection upon ruin (together with the thought of María Zambrano, Walter Benjamin and others) allows us to understand the forms of resistance from which the stories of Ponce Padilla arise.

KEYwORDS: Fragment, story, Ecuador, ruin, triptych, woman, violence, patriarchal, state violence, Gabriela Ponce Padilla.

Gabriela Ponce Padilla (Quito, 1977) es, en el ámbito de las artes escénicas en el Ecuador en lo que lleva de transcurrido el siglo, uno de sus más importantes referentes. Desde su primer montaje, el de Paralelogramo $^{1}$ del escritor vanguardista Gonzalo Escudero (Selección Nacional de Proyectos Escénicos y Escénico-Musicales en la categoría de teatro de texto y experimental del Teatro Sucre, 2007), hasta los últimos trabajos con el colectivo Mitómana (Tazas Rosas de Té, la última obra montada, recibió el Premio Francisco Tobar García del Municipio de Quito al mejor montaje del año 2017 y el de Dramaturgia Inédita del Teatro Sucre,

1. Paralelogramo fue publicada por Escudero en el año 1934; sin embargo, no había sido llevada a escena, hasta que Ponce y su grupo de trabajo de entonces, el colectivo Paralelogramo, en el 2008, deciden montarla en un arriesgado y bien logrado esfuerzo por interpretar y adaptar con recursos contemporáneos un texto que fue catalogado como "obra irrepresentable" por su complejidad vanguardista. 
el mismo año), ha revelado, en su faceta de directora y dramaturga, una capacidad asombrosa para ofrecer historias que se desarrollan en múltiples planos espaciales, temporales y psíquicos; planos que se entretejen al punto que el sentido de montaje teatral adquiere una materialidad radical en la figura del collage. ${ }^{2}$

En las obras escénicas de Ponce Padilla, es a partir del fragmento o de la reconstrucción fragmentada de la memoria que las historias van cobrando sentido y complejidad. Esta estética de lo fragmentario, sabemos, marcará la sensibilidad y el pensamiento del siglo XX, tal como Walter Benjamin anticipara en sus reflexiones en torno al montaje y en su apropiación de esta técnica como poética de su discurso:

Ante la experiencia de la disolución y, sobre todo, de la dispersión de las cosas mismas en el caos de la gran ciudad, el montaje supone un trabajo positivo sobre las ruinas, los harapos, los desperdicios de la historia. Lo que este método le permite a Benjamin es una forma de componer los materiales de una manera en que no se supeditan a una lógica de ordenación jerárquica o a un relato lineal (López 2013, 3-4).

Paralelamente a su labor dramatúrgica, Gabriela se ha dedicado a la escritura narrativa. Actualmente se encuentra escribiendo su primera novela y en 2015 publicó su primer libro de cuentos, Antropofaguitas (ganador del Concurso Nacional de Proyectos para el Fomento y Circulación de las Artes, categoría Artes Literarias, 2013). En esos relatos, muy de la mano de la propuesta estética e intelectual de su trabajo escénico, Ponce plantea algunas relecturas de lo fragmentario a las que quisiera acercarme en primera instancia para pensar cómo se construye un imaginario de las relaciones humanas y el lugar que ocupa la mujer en el entramado social del cambio de siglo. En la misma línea benjaminiana de la recomposición material de la ruina, quisiera proponer que Ponce organiza su primer libro de cuentos. En este ensayo, me interesa acercarme a uno de los relatos del libro para entender qué efectos produce este trabajo en la narración, qué

2. Por ejemplo, su adaptación del cuento de Roberto Bolaño, "Putas asesinas", que tituló Esas putas asesinas, el espectador debía seguir a los actores de una habitación a otra en una casa que había sido adecuada para la obra y que, a partir de una cuidada escenografía, permitía al espectador hacerse una idea de la complejidad psicológica de los personajes. La compartimentación del espacio escénico se correspondía con la psiquis escindida de las protagonistas. 
arroja para el lector en tanto reensamblaje problemático, incompleto, de las historias de sus protagonistas.

Antropofaguitas está compuesto por diez cuentos y nos presenta, en todos los casos, protagonistas mujeres que se enfrentan a la vida cotidiana y que recrean su vida, sus problemas íntimos, sus relaciones personales desde posturas que no solo implican una brutal honestidad, sino que exponen y deconstruyen las instituciones y órganos de control social sobre sus cuerpos, acciones y lenguajes. Esta deconstrucción ocurre a partir de varias estrategias y en varios niveles: en el plano de la estructura familiar, se presenta, por ejemplo, una compleja normalización del incesto; en el plano estético, ocurre la aceptación del kitsch; la conformación identitaria de las protagonistas no arroja personajes cerrados o redondos, sino en constante devenir.

Los tres últimos relatos del libro - "Cadáver en NY", "Caja Negra” y "Tríptico de la Madre"- que, tal como anticipa el título del último de ellos, se estructuran como trípticos: presentan tres momentos o, mejor, tres movimientos que son, en un caso y otro, más o menos continuos. En el primer cuento, la continuidad de las tres partes es evidente ya que estas se desarrollan en el mismo espacio-tiempo y alrededor de los mismos personajes; en los dos últimos, por el contrario, se antologizan tres relatos que convergen por el tratamiento de la estructura ("Caja Negra”) y por el tratamiento de los motivos ("Tríptico de la Madre"). En todos los casos, las protagonistas son mujeres que, en situaciones sociales complejas, a partir de una agencia particular e inesperada, problematizan en torno a ciertos lugares comunes. Así, a la fragmentación quiero sumar la imagen de la ruina como una instancia que nos refiere la destrucción de lo que fue, pero también la resistencia a ser pensado y reconstituido a partir de un otro ajeno o lejano.

En este trabajo quiero concentrarme en el segundo de estos relatos, “Caja Negra”, cuyas historias recrean las muertes de mujeres en diferentes momentos históricos y contextos sociales. El relato como caja negra es el símil utilizado por Ponce para pensar en la necesidad de recrear el contacto comunicacional fuera de su contexto original y lo compleja que resulta esa recreación cuando la voz de la mujer no puede rendir testimonio por sí misma (esto en las dos primeras partes del tríptico), sino que es testimoniada por otros. En el imaginario contemporáneo, a la caja negra como dispositivo que preserva la grabación de los diálogos en la cabina previo a 
un accidente aviatorio, sumamos siempre los despojos humanos, los cuerpos fragmentados, que deben ser leídos desde la medicina forense para poder determinar el cómo y el porqué de la muerte. El lector de los relatos de Ponce también hará su trabajo de pesquisa: se trata menos de determinar, a partir de las pistas que brinda la lectura, las circunstancias o razones de las muertes, pero sí de entender cómo ocurre la práctica antropofágica en esta escritura y la lógica de los afectos.

El lector se enfrenta a la imposibilidad de aprehender el registro de las protagonistas, de comprender sus móviles debido a que el discurso directo de los personajes, cuya palabra sí se reproduce en el cuento, no puede nunca ponerse en el lugar de quien ya no puede hablar. ${ }^{3}$ De este tríptico, cabe entender, suponer, imaginar, aquello que se pierde, para siempre, en la traducción, aquello que no puede ser narrado, pero que debe ser intuido, restituido para la memoria. La estrategia benjaminiana de construir a partir del fragmento se corresponde con el esfuerzo por narrar lo inenarrable o de generar, a partir del nuevo ensamblaje de las piezas ruinosas, una nueva imagen de la mujer en cuestión, en donde más que importar lo que se diga, interesa lo que no se puede decir y permanece latente en los relatos como una fuerza vital, como una señal indistinta de la vida que fue.

“Caja Negra No. 1. Lena” abre con la siguiente cita:

La noche del 10 de febrero fue arrestada en la ciudad de Playas la ciudadana Lena F. de 40 años de edad. La mujer se arrancó un pedazo de lengua y luego la escupió en la oficina del juez penal, como señal de que no hablaría en el caso de la muerte del empresario japonés Keitske $C$. Tsuchia, y en el que estarían involucrados otros dos hombres cuyo paradero se desconoce, uno de los cuales, al parecer, era novio de la sospechosa. Lena F. guardó silencio durante los días que estuvo en prisión. Después del registro de los movimientos de su cuenta de correo electrónico, la policía judicial determinó que quedase en libertad condicional, al no existir pruebas que la inculparan directamente en el crimen, pero a la mañana siguiente, cuando se iba a ejecutar la decisión del juez, la mujer apareció muerta en su celda. La policía alega suicidio, pero hay quienes sostienen que fue asesinato. El caso ha despertado conmoción en la ciudadanía, dada la fortuna y el poder que el

3. A excepción del último relato del tríptico, en donde sí se reproduce la voz de la contrabandista y las demás mujeres que son los personajes centrales de la historia. 
empresario, dueño de una cadena de restaurantes y de una de las agencias de publicidad más importantes del país, ostentaba (Ponce 2015, 153).

A partir de esta cita, cuya fuente no se identifica y que suponemos se trata de una nota de prensa, se reproducirá una serie de correos electrónicos, que, se entiende, se encuentran en la bandeja de entrada del correo de Lena Figueroa. Ninguno de estos correos ha sido escrito por ella, no se lee nunca una respuesta suya. El lector apenas cuenta con la palabra de los otros para ir determinando los acontecimientos. A partir de la lectura de estos correos fechados entre el 5 y el 11 de febrero de 2012; firmados por amigos, familiares y el ex novio de Lena, y transcritos en orden cronológico, podemos sacar las siguientes conclusiones respecto de su vida y de los acontecimientos alrededor de su muerte. Hacia inicios de febrero, Lena comienza a buscar un nuevo trabajo. Es madre de Gastón, a quien una amiga suya se refiere como "tu cachorrito" por lo que se deduce que se trata de un niño pequeño. Lena está separada de quien fue su marido y es padre de Gastón. Ha recibido un diagnóstico médico que no se explicita, cuyo tratamiento implicaría el uso de prótesis. Es pintora y está organizando la exposición de sus óleos y grabados bajo el título de "Muerte y Resurección". En una nota sobre dicha exposición, se hace énfasis en la obsesión monocromática de Lena por el color verde: "es el misterio pero también la clave para entender su obra" (161; cursivas en el original). Trabaja en la agencia de publicidad del empresario japonés Tsuchia -a quien una de sus colegas llama despectivamente "el chino"-. Allí trabajan también A, el novio con el que acaba de romper, y $\mathrm{H}$, el chico de ojos verdes por quien A abandona a Lena y a quien ella misma ha contratado. Según Mónica, la colega del trabajo de Lena, los ojos verdes de H esconden algo; él le genera desconfianza. La hermana de Lena, Ana, ve a A y H cenando juntos, cosa que le molesta mucho. Lena olvida pagar la pensión de la escuela de Gastón. La madre de Lena está vieja y no puede cuidar de sus mascotas. Para la gente de la agencia, Tsuchia está enamorado de $\mathrm{H}$. Piensan que $\mathrm{H}$ es un ambicioso que no conforme con quedarse con $\mathrm{A}$, ahora quiere la fortuna del japonés. El 9 de febrero, su amiga Lorna, invita a Lena a la playa. A le pide que haga terapia. A partir del 9 de febrero no contesta el teléfono. Lena se encuentra sorpresivamente con A y $\mathrm{H}$ en el departamento que ella compartía con el primero. Tsuchia ha sido asesinado y se comenta que se trata de un crimen pasional. 
El color verde es la clave que nos ofrece el cuento que nos permitirá establecer un vínculo entre el pasado y el presente de Lena. En el relato, al menos en dos momentos diferentes -en un correo de Jorge, quien escribe la nota sobre la exposición de Lena, y en una de su madre, Peggy Monina-, se hace alusión a un hecho acontecido hace 16 años: la desaparición de un hombre fundamental en la vida de Lena. El verde, que es el color dominante en su obra pictórica y el color de los ojos de $\mathrm{H}$, parecería irrumpir en el relato como un elemento que distorsiona los límites entre pasado y presente. Es decir, los vuelve similares, angustiosamente similares. Este color es la instancia concreta que refiere cómo la experiencia de la pérdida deviene central en la vida de Lena. La figura paterna ausente, ${ }^{4}$ perdida desde hace 16 años, vuelve a perderse en el presente del cuento, a través de A. Los dos episodios en la vida de Lena, fragmentados, desprovistos de cualquier estructura narrativa por fuera de ellos mismos, escasamente referidos en los correos electrónicos, y vistos a partir de la persistencia del verde, parecerían perder sus especificidades y abrir paso nada más a las afinidades entre ellos, convirtiendo así la pérdida en el signo que marcará la vida y la muerte de la protagonista.

En su ensayo titulado "Aprendiendo de las ruinas", el arquitecto italiano Alberto Ferlenga plantea una reflexión similar para referirse a la constitución de las ciudades en torno a otras ciudades, rasgo que se evidencia en particular cuando estas se hacen ruinas:

4. En el relato no se menciona quién es el personaje que ha desaparecido hace 16 años, pero si damos fe a lo que menciona Jorge en su correo fechado el 6 de febrero de 2012 , queda claro que se trata de una figura masculina trascendental para Lena, unida a ella por su amor a las formas: "Son ya 16 años. Hoy he amanecido pensándolo. A veces escucho como eco su voz en la casa. A ti te pasa? Son los caballos, Lena, los que lo traerán de vuelta? El caballo como dice alguna escritora del siglo pasado, es un animal que se expresa por la forma. Tanto tú como él son amantes de las formas. Son las sombras en las cuencas vacías de tus ojos, de tus autorretratos Lena, su rostro, el rostro imaginado de la muerte? Yo en todos tus cuadros lo veo Lena. En todos tus cuadros" (162). El uso del tiempo presente para referirse a esta figura masculina hace dudar respecto de si su ausencia refiere su muerte o simplemente su desaparición de la vida de Lena. En un correo posterior de su madre, ella le reclama que, con su propia desaparición (el lector sabe que ha viajado a la playa, ya que ha aceptado la invitación de su amiga Lorna), la está haciendo padecer lo mismo que hace 16 años. Este correo parecería confirmar que se trata, no de una muerte, sino de una desaparición. 
La historia de una ciudad, por tanto, nunca es sólo de esa ciudad; es más, podemos decir que todas las ciudades del mundo contribuyen a la formación de cada una de ellas. Esto aparece con especial claridad, como veremos más adelante, cuando las arquitecturas que la caracterizan pierden los signos distintivos de su pertenencia a una época o una geografía, se hacen ruinas, fragmentos sintéticos e irreductibles $\mathrm{y}$, como tales, se sustraen de muchos de los convencionalismos por los que se las reconoce y dilatan en el tiempo y en el espacio sus afinidades (Ferlenga 2013, 19).

En la reconstitución de la vida de Lena, posible a partir de los fragmentos con los que contamos como lectores, los sucesos de su vida, más precisamente los hitos a los que nos hemos referido, parecerían devenir en memorias ruinosas, al estilo de las ciudades que piensa Ferlenga. O, como diría María Zambrano, se trata de memorias que sobreviven a la destrucción, es decir a su muerte, y por lo tanto son lo más viviente de la historia (Zambrano 2012, 250-51). El relato de Ponce no ofrece una respuesta a la pregunta en torno a la muerte de Tsuchia, porque no se trata de una trama policial que se encamina hacia su resolución -aunque así parecería sugerir el contenido de la primera página del relato citado arriba y nos mantengamos en vilo como quien lee un thriller-. "Caja Negra No. 1. Lena" nos permite, finalmente, imaginar el ímpetu de las acciones de una mujer que de un día a otro se enfrenta al desmoronamiento de su vida amorosa y a la cárcel por un crimen que, al parecer, no ha cometido. ${ }^{5}$ De Lena, finalmente sabemos poco; quizás lo único es que muerta no delatará al asesino o los asesinos, y tampoco será la llamada a narrar su propia vida. Su tragedia ha significado entrar en la efusión del verde que representa la figura paterna ausente. Lo que está pasando siempre es esa ausencia. De ahí que calle ante el juez, de ahí que mutile su lengua como la última señal desesperada, amorosa o poética de que guardará para siempre silencio. Aquí quizás cabría pensar en lo que el texto de Ponce tiene de "montaje". Hay cierta fluidez que nos permite ir entendiendo qué es lo que ha sucedido; sin embargo, no hay necesariamente relación entre un correo y otro de los que componen este relato. Lena podría ser otra a los ojos del lector si es que los correos leídos fueran los escritos por ella misma. Ponce monta esta historia de tal modo que el silencio final de Lena ante las autoridades judiciales se corresponda con la ausencia de sus propios correos, de su palabra.

5. O sí. Nada queda muy claro respecto a este asunto. 
Lena como personaje inacabado es en sí mismo una ruina. Sostiene Zambrano:

Mas la contemplación, la visión de la historia misma, trae en algunos momentos la liberación. Porque lo propiamente histórico no es ni el hecho resucitado con todos sus componentes -fantasma de su realidad-, ni tampoco la visión arbitraria que elude el hecho, sino la visión de los hechos en su supervivencia, el sentido que sobrevive tomándolos como cuerpo. No los acontecimientos tal como fueron, sino lo que de ellos ha quedado: su ruina (Zambrano 2012, 250).

Como lectores contemplamos los hechos en su supervivencia. Ponce entiende muy bien que no es posible dar a conocer a Lena como un personaje redondo o acabado; es decir, como una totalidad, porque nuestro tiempo y su lugar de enunciación exigen otras formas, otras estrategias para acercarnos a través de la escritura a una comprensión de la experiencia humana. En la línea del pensamiento del alemán Boris Groys, en este relato se percibe un incesante esfuerzo por hacer entrar a Lena en el flujo, en el permanente movimiento del mundo, abandonando "todo sueño de inmortalidad, verdad eterna, perfección moral, belleza ideal, etc.” (Groys 2016, 19). El acabamiento, la cerrazón no es lo que persiste y mucho menos lo que resiste. La ruina en tanto constante acabamiento es lo que ha resistido, lo que seguirá resistiendo por ser parte de ese permanente movimiento. Y la ruina, como pocos paisajes, nos produce conmoción.

Esta misma recreación ruinosa de la protagonista ocurre en la segunda parte del tríptico, “Caja Negra No. 2. Muerte de una joven anarquista”. Aquí, son varias las voces que, a manera de testimonio, dirán algo sobre la protagonista. Se preservan los nombres y quienes hablan serán identificados por sus iniciales. No se menciona la identidad de ella en ningún momento. Es posible, sin embargo, identificar a cada testigo a partir de su postura respecto de la vida, pero sobre todo de la muerte de la protagonista, una militante de la izquierda armada que muere en circunstancias no esclarecidas o sobre las que no existe consenso: ahí están la compañera de celda durante el cautiverio $(\mathrm{J})$, el político de derecha $(\mathrm{P})$, el compañero de organización $(\mathrm{A})$, la hermana $(\mathrm{H})$, una señora que asegura haber presenciado cómo la mataron $(\mathrm{S})$ y un policía local $(\mathrm{C})$. En un gesto antropofágico, el relato de Ponce echa mano de muchos datos históricos que coinciden con eventos relativos a la lucha armada de la izquierda 
ecuatoriana en la segunda mitad del siglo XX o con datos biográficos de militantes y líderes de esta lucha; ${ }^{6}$ así, los testimonios se convierten en una suerte de variación respecto de ciertos fragmentos de la historia reciente del Ecuador, que nos refiere algunas de las características centrales del proceso revolucionario, así como la represión que el gobierno de León Febres-Cordero ejerció contra los militantes de ¡Alfaro Vive, Carajo! (AVC).

Los testimonios en el relato de Ponce se concentran, como mencioné, en ofrecer diferentes versiones respecto de la muerte de la protagonista. Cada uno de los testigos asegura que la versión que maneja es la verdadera. Así, lo que se dice sobre la vida de la mujer pasa a segundo plano; esto parecería importar menos que las circunstancias de su muerte. No se festeja la vida en el afán de castigar la muerte. La hermana por ejemplo insiste en la necesidad de esclarecer la verdad y de que se haga justicia, a pesar del tiempo transcurrido. Los otros, el político de derecha, el policía, prefieren dejar asentado que los eventos acontecieron en circunstancias legales, siendo que la legalidad a la que apelan va de la mano de la erradicación de los grupos armados en el país. El relato va dibujando una sociedad dividida. Pasados los años, todos los testimoniantes son entrevistados por una persona de la que poco se sabe, pero de la que se dice que está interesada en este asunto concreto y en la historia en general. El interés del interlocutor o la interlocutora anónima podría ser el interés de una persona concreta, pero también recuerda el rol de la Comisión de la Verdad de Ecuador, constituida en el 2007, instalada en el 2008, y cuyo informe fue entregado en el 2010. Los testimonios que conforman el relato de Ponce se asemejan en el tono y contenido a los citados en el informe de dicha comisión en el apartado "Operativo internacional: ejecución extrajudicial de Arturo Jarrín".

6. Por ejemplo, como la protagonista de "Muerte de una joven anarquista", en 1985, Arturo Jarrín, líder de ¡Alfaro Vive, Carajo! (AVC), logra escapar del Penal García Moreno "por un túnel de aproximadamente 300 metros". (Comisión de la Verdad 2010, 357). Disponible en «http://repositorio.dpe.gob.ec/bitstream/39000/1312/5/IE-002.03-2016.pdf). Como Jarrín, la protagonista viaja a Panamá donde una de las versiones sugiere que fue apresada. Asimismo, la muerte de la protagonista de la historia resembla las circunstancias de la muerte de Jarrín, acontecida en 1986.

7. En dicho apartado se lee: "El cuerpo de Arturo Jarrín fue entregado a sus familiares el 27 de octubre de 1986, Miguel Jarrín continúa: 'Tenía innumerables tiros; no los ocho balazos que había dicho la Policía [...]. Innumerables, realmente era indescriptible [...]. Absolutamente en todo el cuerpo, en las piernas, en el tórax, en el rostro, en la boca, en 
Volvamos a la protagonista del relato. El cuerpo entregado a la familia no solo que recibió 30 balazos, sino que también muestra evidentes signos de tortura. Se sabe, además, por el testimonio de su compañera de celda que ambas fueron violadas en los días que precedieron a su asesinato. La violencia machista hace de su cuerpo un despojo que revelará a través del lenguaje y la escritura de la violencia practicada sobre él, que el Estado patriarcal lleva a cabo un ejercicio de basurización de su cuerpo. En su lectura de la basurización simbólica y los discursos autoritarios en el Perú contemporáneo, Rocío Silva Santisteban lee las reflexiones de Daniel Castillo y señala: "No obstante, en su libro Vertederos, Castillo desarrolla la idea de 'basurización del otro' no solo como la basurización de los sujetos, sino, sobre todo, como forma de construir otredades-alteridades funcionales a la lógica hegemónica central” (Silva Santisteban 2008, 63). La evidente estrategia del Estado represor es devolver un cuerpo-basura para debilitar o eliminar la trascendencia de quien en vida resistió todas las formas de opresión posibles. Incluso si la versión de que ella murió a manos de sus propios compañeros de organización, sostenida por A, fuese la verdadera, su muerte acontece bajo la misma lógica de resistencia, esta vez a los manejos inadecuados dentro de la organización guerrillera, que replicará en sus filas un ordenamiento estatal.

¿Cuerpo-basura o cuerpo-ruina? Cuerpo-basura desde la perspectiva del Estado o para Estado represivo y patriarcal, pero cuerpo-ruina desde la perspectiva de la resistencia. El ser ruina de ese cuerpo no implica que el peligro desaparece; hay más bien una hiperconciencia de la violencia del decurso histórico. Sostiene Ruiz de Samaniego:

la cabeza...". En el mismo protocolo de autopsia se pueden advertir signos que evidencian maltratos que no corresponden con las circunstancias de su muerte aducidas por la Policía. En el documento de autopsia se señalan golpes y excoriaciones en todo su cuerpo, incluso en sus testículos" (Comisión de la Verdad, 380). En el relato de Ponce, encontramos el siguiente fragmento del testimonio de la hermana de la protagonista: "Sí, parece que la traen en un avión dormida, esa es la versión a la que finalmente llegamos, porque ellos nunca la aceptaron. Parece que allá no podían matarla, entonces la durmieron y la mataron después de torturarla algunos días en los calabozos que tenían en la Mariscal. La llevaron por la zona del aeropuerto ya estando inconsciente y le dispararon en plena calle un sin fin de balazos, no me pregunte para qué. Le tenían tanto miedo que decidieron dispararle 30 balazos" (Ponce, 195). 
El paradójico vigor de la ruina es testimonio no sólo de la soberbia de los hombres, quienes creen erigir algo permanentemente en medio de la finitud del mundo; sino, aún más, de la propia fragilidad de una existencia sometida fatalmente a la cruel inconsciencia de la naturaleza y al propio carácter destructivo del decurso histórico. Ante la ruina la mirada ve, con estupor e impotencia -tal como lo siente la alegoría-, que la vida toda se encuentra en peligro, dispuesta siempre bajo el signo de la destrucción y el despojo (Ruiz de Samaniego 2013, 212).

Esta hiperconciencia abre paso a la identificación entre la historia personal de la protagonista y la historia misma enmarcada en lo que Zambrano llama "supervivencia, no ya de lo que fue, sino de lo que no alcanzó a ser" (Zambrano 2012, 251). La muerte de la militante suspende la negatividad (en términos dialécticos), pero no la elimina. Lo que no alcanzó a ser, digamos que el anarquismo como religión, "cuyo ritual es la vida" (Ponce, 205; cursivas en el original), persiste en el tiempo como una fuerza latente que sostiene toda forma de rebelión y resistencia en el tiempo del cotidiano y en el tiempo histórico. En el cuento de Ponce, hay al menos dos testimonios que liberan al cuerpo de la protagonista de esa carga sígnica otorgada por las fuerzas estatales al cuerpo de la mujer. Sostiene J, su compañera de celda hacia el final del cuento: "Era impresionante por su coraje y por su belleza. Era como de otro tiempo su belleza, eso sí. Era como antigua su belleza. Y además era una belleza que se contagiaba. Todo a su alrededor se embellecía. Hasta los chapas. En serio. No se ría, era verdad. Se murió de lo linda que era creo yo" (204). Esa belleza que parecería ser física, quisiera leerla aquí como una belleza del espíritu, que no muere, que no decae. Al embellecerlo todo (inclusive a los policías que la torturan) no se está apelando necesariamente o no solamente a una experiencia estética, sino al hecho de que su existencia signada por el anarquismo está insuflando esperanza en todas las instancias de la vida social, del presente y del futuro. La belleza de la protagonista descrita por J, entonces, tiene un potencial alegórico que, en la línea de Benjamin, puede ser leído como la necesidad de tomar conciencia críticamente y no pasar por alto cuando ese fragmento del pasado ha dado en encontrarse con nuestro presente o con otros presentes (Benjamin 2009, 71).

El tríptico cierra con “Caja Negra No. 3. La contrabandista”. Este relato se estructura a partir de la transcripción de lo que parece ser una escucha ilegal en la casa donde una contrabandista vive con su hija y que 
es su centro de operaciones para el contrabando de pelo, explosivos y pistolas. Este se desmarca de los dos primeros relatos porque aquí las mujeres hablan por ellas mismas. No ofrecen un testimonio y en ese sentido la búsqueda de la verdad como objetivo, de plano, se anula. Queda arriesgar lecturas posibles en torno a la dinámica de estas mujeres que ocurre en un período de tiempo de 12 minutos entre que la Madre llega a la casa, encuentra a su hija y a una de sus secuaces, Sofía, en el baño, hasta que se oye el sonido de una explosión cuando los policías vuelven a tocar la puerta de casa para continuar con las averiguaciones y posiblemente una revisión del departamento a causa de los acontecimientos de las últimas semanas: asaltos a mujeres por su pelo, robo de armas y de una bicicleta, la presencia de un gato en el condominio a pesar de estar prohibidas las mascotas.

A pesar de la insistencia de la policía, las contrabandistas tienen la oportunidad de huir del departamento. Tratan de llevarse la mayor parte de la mercancía que ahí quedaba y huyen por la puerta trasera del edificio. La explosión final, que no se sabe si acaba con la vida de las mujeres o de los policías o los lastima, parecería ocurrir como el develamiento de un misterio ulterior. Final inesperado para una historia en donde se realiza un contrabando con un objeto inesperado, asimismo: el pelo de mujeres. La disposición tipográfica de este relato en donde se señala la hora de cada parlamento hasta con décimas de segundo, parecería al final explicarse como el tiempo del dispositivo cronométrico adjunto a una bomba.

El carácter fuerte de la contrabandista, que recibe el nombre de Madre en el relato, se contrapone al de las demás mujeres: Claudia, la hija, de semblante mucho más tranquilo con quien la relación es de absoluto dominio; Sofía, su secuaz, que en el relato se presenta como una mujer cuyo discurso parece girar en buena medida alrededor de su sexualidad y a quien una madre de familia del condominio busca acusándola de acosar a su hija, y Moni, la otra contrabandista, que ha llegado a retirar el gato de Sofía. La Madre es una mujer dura, deja todo el tiempo claro que su rol es el de líder y que no acepta ningún tipo de exceso de parte de las demás. Mientras las otras mujeres buscan desviar el discurso hacia otras zonas, ella vuelve siempre sobre el asunto del contrabando y termina por imponerlo.

Los objetos con los que trabaja esta banda son absolutamente disímiles; no hay una lógica detrás; parece casi ridículo (aunque exista en realidad este tipo de contrabando) que se dediquen al tráfico de pelo. De pronto, en el relato, se nombran elementos que intensifican la sensa- 
ción de absurdo o al menos dejan claro que aquello que escuchamos no nos refiere un panorama completo de la vida de estas mujeres: como, por ejemplo, cuando tocan el timbre y no saben quién puede ser, Claudia se cuestiona si quizás han llegado a recoger los explosivos, el pelo o las traducciones. No se sabe a qué traducciones se refiere y no se vuelve a hablar de ellas en lo que queda del relato. Las cosas extrañas que acontecen, o los elementos que rompen las series o numeraciones, no se explican, están ahí como elementos disruptores de cualquier orden o lógica y esa parece ser su única función. El final vertiginoso del relato, en el que las mujeres están preparándose para abandonar el edificio, los disruptores parecen acumularse: guardan los explosivos sobrantes en la refri; la Madre a toda costa ha de llevarse un cuadro; cuando lanzan el pelo que quedaba por la ventana, la Madre les recuerda que la cierren para que no se les metan los ladrones. Luego, la explosión y, con ella, el fin de la historia, el silencio, las nuevas ruinas. Esta vez, la ruina ya no es la mujer, su relato o su despojo, sino el departamento mismo. Suponemos que la explosión acentúa el aspecto ruinoso de los objetos personales que seguramente quedaron ahí, o las herramientas de trabajo o el contrabando sobrante. La explosión final arrasa con la huella de las mujeres en ese espacio, pero deviene huella asimismo de su paso por ahí. ${ }^{8}$ ¿Cabe leer la explosión como triunfo, como derrota, como la instancia física del sinsentido?

"Caja Negra” nos plantea preguntas sobre el pasado, sobre su reconstrucción, $y$, en última instancia, sobre cómo ocurre a partir suyo el acto de narrar. Narramos como antropófagas, sería una de las respuestas posibles. En este relato de Gabriela Ponce Padilla, se acumulan los fragmentos y las ruinas como un ejercicio de corte benjaminiano que asegura en la mirada hacia atrás la resistencia respecto del desesperante e inminente paso del progreso. La caja negra es la huella de la destrucción, el sobrante del accidente, contiene la grabación de las voces en el instante inmediatamente anterior a la muerte. ¿Qué hacer con las voces que ya no están, sobre todo cuando se tiene la certeza de que los mejores murieron? ¿Cómo lidiar con la angustia, con la muerte? ¿Qué hacer con las ruinas? En el ensa-

8. Sostiene Zambrano: "También las cosas gastadas muestran el paso del tiempo y en el caso de un objeto usado por el hombre algo más: la huella, siempre misteriosa, de una vida humana grabada en su materia. Un cepillo usado, un zapato viejo, un traje raído, casi llegan a alcanzar la categoría de ruina. Porque ruina es solamente la traza de algo humano vencido y luego vencedor del paso del tiempo" (253). 
yo de Ferlenga que cité anteriormente, el arquitecto italiano sostiene que las ruinas usadas por los habitantes de las ciudades suelen preservarse bastante bien con el paso del tiempo. La permanencia in situ de la población, en muchos casos, funciona mejor que el uso exclusivamente museístico de esas ruinas. Ahí justamente, me parece, se instala la narración antropófoga de Ponce: los relatos de las vidas de estas mujeres no nos muestran seres terminados, completos, no nos regalan piezas de museo, sino personajes que nuestros ojos y manos de lectores aprender a habitar indistintamente. Tragamos estos relatos para hacerlos carne de nuestra carne. *

\section{Bibliografía}

Barja, Juan, y César Rendueles, editores. 2013. Mundo escrito. 13 derivas desde Walter Benjamin. Madrid: Círculo de Bellas Artes.

Benjamin, Walter. 2009. "Eduard Fuch, coleccionista e historiador". En Obras II, 2. Madrid: Abada. 68-108.

Comisión de la Verdad. 2010. Informe de la Comisión de la Vedad Ecuador 2010. Quito. Disponible en http://repositorio.dpe.gob.ec/bitstream/39000/13 12/5/IE-002.03-2016.pdf .

Ferlenga, Alberto. 2013. “Aprendiendo de las ruinas”. En Antonio Tejedor Cabrera y Mauro Marzo, editores. Sevilla: Universidad Internacional de Andalucía.

Groys, Boris. 2016. "Entrar al flujo". En Arte en flujo. Buenos Aires: Caja Negra. 17-31.

López, Nicolás. 2013. "El principio del montaje en Walter Benjamin”. Revista Lindes. Estudios Sociales del Arte y la Cultura 6: 1-13. Disponible en «http://www. revistalindes.com.ar/contenido/numero6/nro6_art_lopez.pdf). Consulta: 13 febrero 2018.

Ponce Padilla, Gabriela. 2015. "Caja Negra”. En Antropofaguitas. Quito: Ministerio de Cultura y Patrimonio del Ecuador. 153-223.

Ruiz de Samaniego, Alberto. 2013. "De ruinas: Piranesi, Benjamin, Smithson (una familia)".

Silva Santisteban, Rocío. 2008. El factor asco. Basurización simbólica y discursos autoritarios en el Perú contemporáneo. Lima: Red para el Desarrollo de las Ciencias Sociales en el Perú.

Zambrano, María. 2012. El hombre y lo divino. México D. F.: Fondo de Cultura Económica. 